\title{
Mutational analysis of multiple lung cancers: Discrimination between primary and metastatic lung cancers by genomic profile
}

\author{
Taichiro Goto ${ }^{1, *}$, Yosuke Hirotsu ${ }^{2, *}$, Hitoshi Mochizuki², Takahiro Nakagomi ${ }^{1}$, Daichi

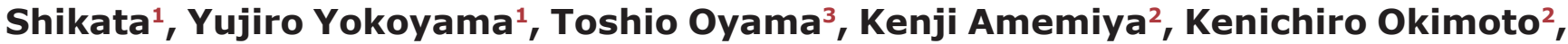 \\ Masao Omata ${ }^{2,4}$ \\ ${ }^{1}$ Lung Cancer and Respiratory Disease Center, Yamanashi Central Hospital, Yamanashi, Japan \\ ${ }^{2}$ Genome Analysis Center, Yamanashi Central Hospital, Yamanashi, Japan \\ ${ }^{3}$ Department of Pathology, Yamanashi Central Hospital, Yamanashi, Japan \\ ${ }^{4}$ University of Tokyo, Tokyo, Japan \\ *These authors contributed equally to this work
}

Correspondence to: Taichiro Goto, email: taichiro@1997.jukuin.keio.ac.jp

Keywords: lung cancer, multiple cancers, metastasis, mutation, next-generation sequencing

Received: November 30, $2016 \quad$ Accepted: February 28, $2017 \quad$ Published: March 10, 2017

Copyright: Goto et al. This is an open-access article distributed under the terms of the Creative Commons Attribution License (CC-BY), which permits unrestricted use, distribution, and reproduction in any medium, provided the original author and source are credited.

\section{ABSTRACT}

In cases of multiple lung cancers, individual tumors may represent either a primary lung cancer or both primary and metastatic lung cancers. Treatment selection varies depending on such features, and this discrimination is critically important in predicting prognosis. The present study was undertaken to determine the efficacy and validity of mutation analysis as a means of determining whether multiple lung cancers are primary or metastatic in nature. The study involved 12 patients who underwent surgery in our department for multiple lung cancers between July 2014 and March 2016. Tumor cells were collected from formalin-fixed paraffin-embedded tissues of the primary lesions by using laser capture microdissection, and targeted sequencing of 53 lung cancer-related genes was performed. In surgically treated patients with multiple lung cancers, the driver mutation profile differed among the individual tumors. Meanwhile, in a case of a solitary lung tumor that appeared after surgery for double primary lung cancers, gene mutation analysis using a bronchoscopic biopsy sample revealed a gene mutation profile consistent with the surgically resected specimen, thus demonstrating that the tumor in this case was metastatic. In cases of multiple lung cancers, the comparison of driver mutation profiles clarifies the clonal origin of the tumors and enables discrimination between primary and metastatic tumors.

\section{INTRODUCTION}

In cases of synchronous or metachronous multiple cancers, individual tumors may be comprised of either a primary lung cancer or both primary and metastatic lung cancers. Treatment selection is dependent on such features. Usually, in cases of multiple lung cancers, whether a tumor is metastatic or primary can be judged on the basis of its clinical course, diagnostic imaging findings and/or pathology. In particular, when diagnostic imaging findings are assessed, a tumor is suspected of being metastatic if multiple small nodules appear simultaneously and assume a round and smooth-surfaced form. In that case, primary lung cancer is usually large, while its metastatic sites within the lung are often smaller in comparison. Thus, the coexistence of a large tumor with multiple smaller nodules within the lung strongly suggests metastasis. In cases of tumors with distant lymph node metastasis, multiple lung lesions are often diagnosed as intrapulmonary metastasis. In contrast, if individual tumors constituting multiple lung cancers are pathologically different from each other in terms of histological type and/or cellular atypism, synchronous onset of multiple primary cancers is deemed likely. However, there are no specific clinical or radiologic features that can be used to confidently distinguish multiple primary cancers from intrapulmonary metastases in all cases, and the differential diagnosis is at times perplexing in the clinical setting. Their different 
biological activities may be responsible for prognostic differences, and patients with intrapulmonary metastasis tend to have a poor prognosis. Therefore, it is important to find ways to identify them by exploring new practical techniques and markers. Certain case studies have been published involving the diagnosis of multiple lung cancers based on the analysis of specific mutations such as TP53, $K R A S$ and EGFR [1-6]. However, the analysis used in these studies does not cover all lung cancer-related gene mutations, so this approach obviously does not always allow for precise judgment. There may also be cases in which such an approach is difficult to apply clinically. As both a more precise and clinically applicable method, we undertook comprehensive mutation analysis with targeted deep sequencing and evaluated the possibility of identifying the clonality of individual lung cancers by using their mutations as a diagnostic marker. Furthermore, we evaluated the efficacy and broad clinical utility of this novel method from the standpoint of understanding the pathology and selecting the most appropriate treatment.

\section{RESULTS}

\section{Patient characteristics}

The 12 patients were divided into groups by the following characteristics (Table 1): 10 males, 2 female; 2 current smokers, 8 former smokers, 2 never smoker; pathological stage IA (4), IB (5), IIB (2) and IV (1). The patients' age ranged between 54 and 82 (mean \pm SD 70.6 $\pm 7.9)$ years. The maximum diameter of the tumors ranged between 8 and $60($ mean \pm SD: $22.8 \pm 12.7)$ millimeters.

\section{Targeted sequencing identified somatic mutations in lung cancers}

We examined 24 surgically resected tumors from 12 patients for targeted sequencing, with their buffy coat samples utilized as normal controls. The mean coverage depth was 1063-fold for tumor samples (range: 273-2471) and 1192-fold for buffy coat samples (range: 472-2428). Sequence analyses identified 102 somatic mutations with an allele fraction $\geq 1 \%$ from 24 tumors (1-12 mutations per tumor) (Figure 1). Among these, 57 mutations (56\%) were present at an allele fraction $\geq 20 \%$ (Table 2). In each patient, the gene, amino-acid substitution and nucleotide changes of these somatic mutations within the individual tumors constituting the multiple lung cancers lacked consistency (Table 2 and Figure 1). Thus, there was no overlap of mutations among the individual lung cancers detected in any patient. This finding demonstrated that the multiple lung cancers in each case were synchronously developing primary lung cancers (Table 2 and Figure 1). Three representative cases are presented below in detail.

\section{Case presentations}

Case 1: A 57 year-old man came to our department after abnormalities were detected on a chest radiograph taken in the course of a health checkup. A chest computed tomography (CT) revealed a tumor (60 $\mathrm{mm}$ in diameter) in the right middle and lower lobes (Figure 2A). Bronchoscopy yielded a diagnosis of squamous cell carcinoma. Preoperative CT and positron emission tomography (PET) showed a small nodule in the right upper lobe, which was interpreted by radiologists as intrapulmonary tumor metastasis (Figure 2B). Based on the assumption that the small nodule in the right upper lobe might be primary, surgery was performed. Right upper lobe wedge resection was performed and intraoperative pathological examination yielded a diagnosis of keratinizing squamous cell carcinoma. However, pathological distinction between the primary and metastatic tumors was deemed difficult, so the operation continued and middle and lower lobectomy was performed. The postoperative pathological examination rated both the upper lobe tumor and the middle/lower lobe tumor as keratinizing squamous cell carcinomas (Figure $2 \mathrm{~A}$ and $2 \mathrm{~B}$ ), without any definitive judgment as to whether either tumor was primary or metastatic in nature. Upon targeted deep sequencing, the mutations in the upper lobe tumor involved NF1, NRAS, ARID2, NFE2L2, EP300 and $K M T 2 D$, while the mutations in the middle/lower lobe tumor involved SMARCA4, TP53, FOXP2, RIT1 and $M G A$ (Table 2 and Figure 1). Thus, the mutation pattern differed completely between these two tumors, allowing for the determination of double primary lung cancers. Postoperative adjuvant chemotherapy was administered in this case. To date, the patient has had no recurrence for the 12 months that have elapsed since surgery.

Case 2: A 67 year-old man came to our department after a chest X-ray revealed an abnormal shadow. Chest CT showed ground-glass opacity (20 $\mathrm{mm}$ in diameter) in right $\mathrm{S} 1$ as well as a nodular shadow $(15 \mathrm{~mm}$ in diameter) in right S6 (Figure 3A and 3B). Lung cancer was strongly suspected and the patient underwent surgery. When the chest was opened, a small volume of pleural effusion was observed, and adenocarcinoma cells were found by pleural lavage cytology. Thus, right upper lobectomy and partial resection of the right lower lobe were performed. Pathologically, the tumor in S1 was classified as a lepidic adenocarcinoma, pleural-invasion negative, and the tumor in S6 was classified as a solid adenocarcinoma, pleuralinvasion positive. Because of slight differences in the histological findings of the two tumors (Figure $3 \mathrm{~A}$ and $3 \mathrm{~B}$ ), double primary cancers were suspected. Upon targeted deep sequencing, significant mutations in the S1 tumor involved $K R A S$, while the significant mutations in the S6 tumor involved TP53, MAP2K1 and FOXP2 (Table 2 
Table 1: Patient characteristics

\begin{tabular}{|c|c|c|c|c|c|c|c|c|c|}
\hline & Age & Gender & Smoking & $\begin{array}{c}\text { Tumor } \\
\text { location }\end{array}$ & Operative procedure & $\begin{array}{l}\text { Size } \\
(\mathrm{mm})\end{array}$ & pTNM & p-stage & Histopathology \\
\hline 1 & 57 & male & former & $\mathrm{rS} 6, \mathrm{rS} 2$ & $\begin{array}{l}\text { middle and lower lobectomy, } \\
\text { wedge resection }\end{array}$ & 60,15 & $\mathrm{~T} 2 \mathrm{bN} 1 \mathrm{M} 0$ & IIB & $\begin{array}{l}\text { (1) ker Sq, ly1, v1, p11, (2) ker Sq, } \\
\text { ly0, v1, pl2 }\end{array}$ \\
\hline 2 & 67 & male & former & $\mathrm{rS} 1, \mathrm{rS} 6$ & $\begin{array}{l}\text { right upper lobectomy, wedge } \\
\text { resection }\end{array}$ & 20,15 & T2aN0M1a & IV & $\begin{array}{c}\text { (1) inv Ad, lepid, ly0, v0, pl0, (2) inv } \\
\text { Ad, solid, ly0, v0, pl1 }\end{array}$ \\
\hline 3 & 74 & male & current & $\mathrm{rS} 2, \mathrm{rS} 3$ & middle and upper lobectomy & 35,35 & T2aN0M0 & IB & $\begin{array}{c}\text { (1) inv Ad, solid, ly1, v0, pl3, (2) inv } \\
\text { Ad, papillary, ly0, v1, pl0 }\end{array}$ \\
\hline 4 & 82 & male & former & $\mathrm{rS} 10, \mathrm{rS} 6$ & right lower lobectomy & 25,20 & T1bN0M0 & IA & $\begin{array}{l}\text { (1) inv Ad, lepid, ly0, v0, pl0, (2) } \\
\text { inv Ad, lepid, ly0, v0, pl0 }\end{array}$ \\
\hline 5 & 54 & male & former & $\begin{array}{c}\mathrm{rS3} \\
\text { leftS1+2 }\end{array}$ & $\begin{array}{l}\text { right upper lobectomy, left } \\
\text { upper division segmentectomy }\end{array}$ & 32,17 & T2aN0M0 & IB & $\begin{array}{l}\text { (1) inv Ad, lepid, ly1, v1, pl0, (2) } \\
\text { MIA, ly0, v0, pl0 }\end{array}$ \\
\hline 6 & 73 & female & never & $\mathrm{rS} 10, \mathrm{rS} 4$ & partial resections & 11,8 & T1aN0M0 & IA & $\begin{array}{l}\text { (1) inv muc Ad, ly0, v0, pl0 (2) } \\
\text { MIA, ly0, v0, pl0 }\end{array}$ \\
\hline 7 & 72 & male & former & leftS1+2 & $\begin{array}{l}\text { left upper division } \\
\text { segmentectomy }\end{array}$ & 15,10 & T1aN0M0 & IA & $\begin{array}{c}\text { (1) AIS, , ly0, v0, pl0, (2) MIA, } \\
\text { ly0, v0, pl0 }\end{array}$ \\
\hline 8 & 73 & male & current & rS2, leftS8 & $\begin{array}{l}\text { right wedge resection, left lower } \\
\text { lobectomy }\end{array}$ & 20,18 & T2aN0M0 & IB & $\begin{array}{l}\text { (1) inv Ad, papillary, ly0, v0, p10, (2) } \\
\text { small, ly0, v1, p12 }\end{array}$ \\
\hline 9 & 67 & female & never & rS3, leftS9 & $\begin{array}{l}\text { right upper lobectomy, left } \\
\text { lower lobectomy }\end{array}$ & 20,25 & T2aN0M0 & IB & $\begin{array}{c}\text { (1) inv Ad, lepid, ly0, v0, p10, (2) inv } \\
\text { Ad, papillary, ly0, v0, pl1 }\end{array}$ \\
\hline 10 & 73 & male & former & $\begin{array}{l}\text { leftS3, } \\
\text { leftS8 }\end{array}$ & left pneumonectomy & 33,8 & T2aN0M0 & IB & $\begin{array}{c}\text { (1) ker Sq, ly0, v1, p10, (2) AIS, ly0, } \\
\text { v0, p10 }\end{array}$ \\
\hline 11 & 77 & male & former & $\begin{array}{l}\text { leftS6, } \\
\text { leftS10 }\end{array}$ & left lower lobectomy & 52,20 & T2bN1M0 & IIB & $\begin{array}{c}\text { (1) ker Sq, ly1, v1, pl1, (2) small, ly0, } \\
\text { v1, p12 }\end{array}$ \\
\hline 12 & 78 & male & former & $\mathrm{rS} 2, \mathrm{rS} 3$ & right upper lobectomy & 17,15 & T1aN0M0 & IA & $\begin{array}{l}\text { (1) MIA, ly0, v0, pl0, (2) inv Ad, } \\
\text { acinar, ly0, v0, pl0 }\end{array}$ \\
\hline
\end{tabular}

S, segment; ker Sq, keratinizing squamous cell carcinoma; Ad, adenocarcinoma; inv, invasive; muc, mucinous; MIA, microinvasive adenocarcinoma; AIS, adenocarcinoma in situ.

and Figure 1). Thus, the mutation profiles differed completely between the two tumors, supporting the view that these were double primary cancers. Postoperative chemotherapy was administered for this stage IV lung cancer. The patient has had no recurrence for 14 months to date since the surgery.

Case 3: A 74-year-old man visited our department for further examination of abnormalities on a routine chest radiograph. Chest CT revealed two tumors $(35 \mathrm{~mm}$ in diameter) in the right upper lobe (Figure 4A and 4B). Bronchoscopy indicated adenocarcinoma. Right upper lobectomy was performed based on the diagnosis of double primary lung cancers (Figure 4C). Pathologically, the segment 2 (S2) tumor was rated as a solid adenocarcinoma and the $\mathrm{S} 3$ tumor as a papillary adenocarcinoma (Figure $4 \mathrm{~A}$ and $4 \mathrm{~B}$ ), thus suggesting that this was likely a case of double primary cancers. On targeted deep sequencing, mutations in the S2 tumor involved TP53 (chr17: 7577157, $\mathrm{T} \rightarrow \mathrm{A}$ splice site mutation), while mutations in the $\mathrm{S} 3$ tumor involved NF1, CDKN2A and TP53 (chr17: 7578556, $\mathrm{T} \rightarrow \mathrm{C}$ splice site mutation) (Table 2 and Figure 1). TP53 mutations were seen in both segments, but they differed in nucleotide position and variance. Such a complete difference in mutation patterns supported the judgment of double primary cancers. During outpatient follow-up, a new tumor developed in the left $\mathrm{S} 1+2$ at 6 months after surgery (Figure 4D). Bronchoscopy revealed it to be an adenocarcinoma (Figure 4E), but was unable to determine whether this new tumor was primary or metastatic. Therefore, targeted deep sequencing was performed with bronchoscopically biopsied specimens to compare the mutations with those found in the previously resected specimens. Upon performing this comparison, the mutation in the left $\mathrm{S} 1+2$ tumor involved $T P 53, N F 1$ and $C D K N 2 A$, identical to that of the right S3 tumor (Figure 4F). Furthermore, the nucleotide position and variance of the mutation in $\mathrm{S} 1+2$ were entirely consistent with those in S3 (Table 2). Thus, a diagnosis of solitary, contralateral, intrapulmonary metastasis was made, and systemic chemotherapy was started. 10 months after the surgery, multiple intrapulmonary, liver and brain metastases developed and then a swelling skin lesion appeared on the left iliac region, which was biopsied to be a metastatic adenocarcinoma histologically. From the view of the mutation profiles, the predominant mutation in the skin lesion involved TP53, $N F 1$ and $C D K N 2 A$, identical to that of the right $\mathrm{S} 3$ adenocarcinoma, not $\mathrm{S} 2$ adenocarcinoma (Figure 5). According to the cluster analysis, the left lung tumor and the skin lesion cluster together genomically with the right $\mathrm{S} 3$ cancer and those clusters segregate away from the right S2 cancer (Figure 5). 16 months after the surgery, the patient died of his cancer due to respiratory failure. 


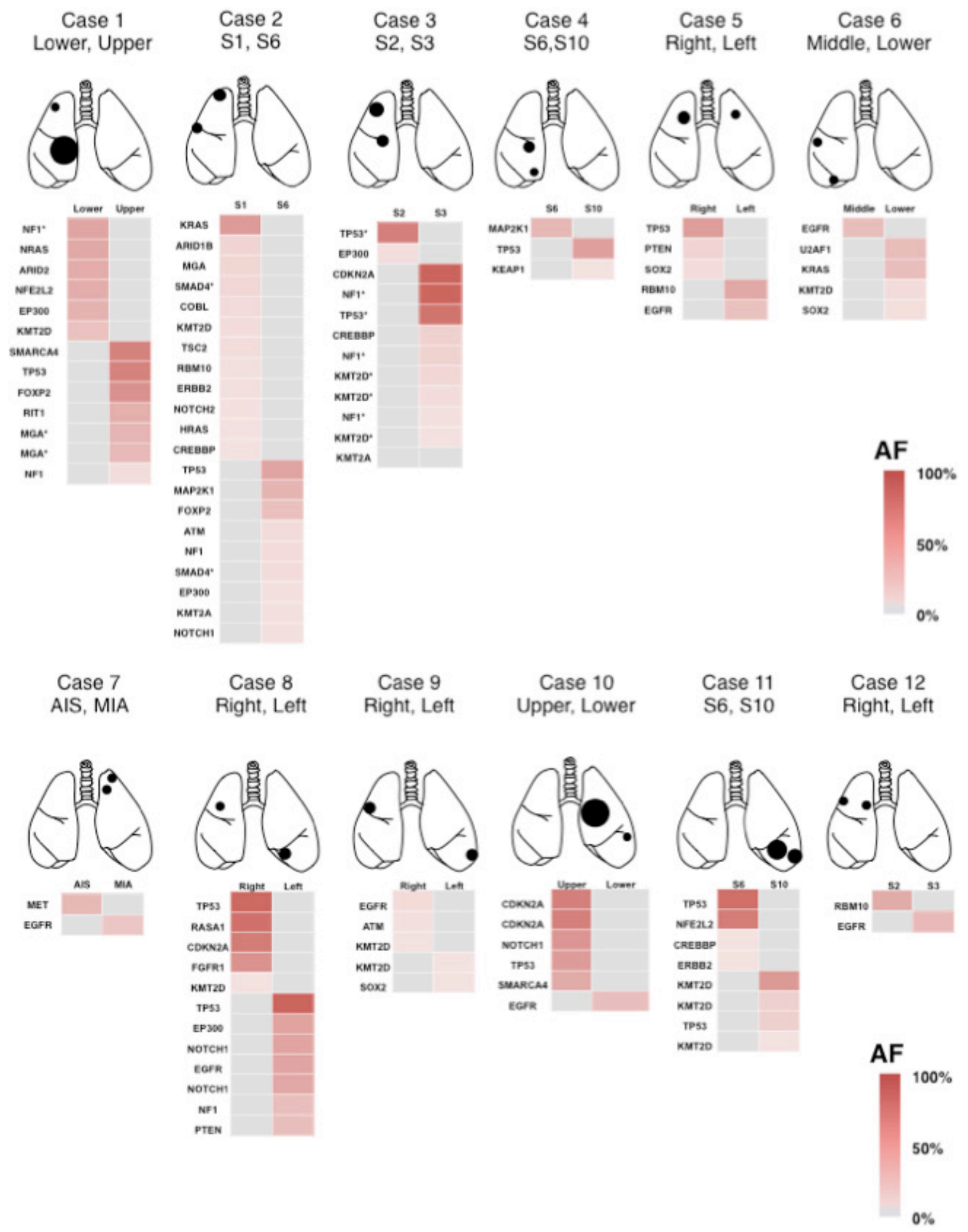

Figure 1: Heat map of the gene mutations in 12 patients with multiple lung cancers. This map visualizes the gene mutations of each cancer, including those with an allele fraction below $20 \%$. Two lung cancers in each patient were characterized by different gene mutations, and all of the cases were judged as having double primary lung cancers. Asterisk $\left(*^{*}\right)$ indicates the different mutations in the same gene. S, segment; AIS, adenocarcinoma in situ; MIA, microinvasive adenocarcinoma; AF allele fraction. 
Table 2: Mutation analysis of the multiple lung cancers

\begin{tabular}{|c|c|c|c|c|c|c|c|c|}
\hline Case & Tumor location & Histology & Gene & Mutation & Position & Ref. & Tumor var. & AF \\
\hline \multirow[t]{12}{*}{1} & & & NF1 & p.Tyr2476Phe & chr17:29677306 & A & $\mathrm{T}$ & $50 \%$ \\
\hline & & & NRAS & p.Gln61Lys & chr1:115256528 & G & $\mathrm{T}$ & $49 \%$ \\
\hline & right S6 & SCC & ARID2 & p.Thr219Ser & chr12:46215221 & $\mathrm{C}$ & G & $45 \%$ \\
\hline & & & NFE2L2 & p.Asp178His & chr2:178097182 & $\mathrm{C}$ & G & $44 \%$ \\
\hline & & & EP300 & p.Ser2328fs & chr22:41574692 & GTCCT & GTCCCT & $41 \%$ \\
\hline & & & KMT2D & p.Arg2830Ter & chr12:49432651 & G & A & $29 \%$ \\
\hline & & & SMARCA4 & p.Glu371Ter & chr19:11098593 & G & $\mathrm{T}$ & $75 \%$ \\
\hline & & & TP53 & p.Arg248Trp & $\operatorname{chr17:7577538}$ & G & A & $74 \%$ \\
\hline & right S2 & $\mathrm{SCC}$ & FOXP2 & p.Pro277Leu & chr7:114271740 & $\mathrm{C}$ & $\mathrm{T}$ & $64 \%$ \\
\hline & & & RIT1 & p.Thr70Ser & chr1:155880247 & $\mathrm{T}$ & A & $43 \%$ \\
\hline & & & MGA & p.Asp339His & chr15:41962107 & $\mathrm{G}$ & $\mathrm{C}$ & $39 \%$ \\
\hline & & & MGA & p.Glu1249Lys & $\operatorname{chr15:42021449}$ & G & A & $35 \%$ \\
\hline \multirow[t]{4}{*}{2} & right $\mathrm{S} 1$ & Adeno & KRAS & p.Gly12Ala & chr12:25398284 & $\mathrm{C}$ & G & $58 \%$ \\
\hline & & & TP53 & p.Cys238Phe & $\operatorname{chr17:7577108}$ & $\mathrm{C}$ & A & $51 \%$ \\
\hline & right S6 & Adeno & MAP23K1 & p.Lys57Asn & chr15:66727455 & G & $\mathrm{T}$ & $39 \%$ \\
\hline & & & FOXP2 & p.Gln250Lys & chr7:114271658 & $\mathrm{C}$ & A & $31 \%$ \\
\hline \multirow[t]{6}{*}{3} & & & NF1 & p.Glu2358fs & chr17:29670037 & AAGTAT & A & $95 \%$ \\
\hline & right S3 & Adeno & CDKN2A & splicesite_3 & chr9:21970900 & $\mathrm{C}$ & $\mathrm{T}$ & $95 \%$ \\
\hline & & & TP53 & splicesite_5 & $\operatorname{chr17:7578556}$ & $\mathrm{T}$ & $\mathrm{C}$ & $85 \%$ \\
\hline & & & TP53 & splicesite_5 & chr17:7578556 & $\mathbf{T}$ & $\mathrm{C}$ & $88 \%$ \\
\hline & left $S 1+2, b x$ & Adeno & NF1 & p.Glu2358fs & $\operatorname{chr17:29670037}$ & AAGTAT & $\mathbf{A}$ & $88 \%$ \\
\hline & & & CDKN2A & splicesite_3 & chr9:21970900 & $\mathrm{C}$ & $\mathbf{T}$ & $83 \%$ \\
\hline \multirow[t]{2}{*}{4} & right S6 & Adeno & MAP2K1 & p.Ser222Thr & chr15:66774188 & $\mathrm{T}$ & A & $37 \%$ \\
\hline & right S10 & Adeno & TP53 & p.Val173Glu & $\operatorname{chr17:7578368}$ & A & $\mathrm{T}$ & $54 \%$ \\
\hline \multirow[t]{3}{*}{5} & left $\mathrm{S} 1+2$ & Adeno & RBM10 & p.Glu721Ter & chrX:47044469 & G & $\mathrm{T}$ & $48 \%$ \\
\hline & & & EGFR & p.Leu858Arg & chr7:55259514 & $\mathrm{T}$ & G & $30 \%$ \\
\hline & right $\mathrm{S} 1$ & Adeno & TP53 & p.Arg280Thr & $\operatorname{chr17:7577094}$ & $\mathrm{C}$ & G & $56 \%$ \\
\hline \multirow[t]{3}{*}{6} & right $\mathrm{S} 10$ & Adeno & U2AF1 & p.Ser34Phe & chr21:44524456 & G & $\mathrm{G} / \mathrm{A}$ & $33 \%$ \\
\hline & & & KRAS & p.Gly12Asp & chr12:25398280 & GCCAC & GCCAC/GCCAT & $32 \%$ \\
\hline & right S4 & Adeno & EGFR & p.E746-R748 del & $\operatorname{chr} 7: 55242478$ & G & $\mathrm{G} / \mathrm{C}$ & $31 \%$ \\
\hline \multirow[t]{2}{*}{7} & left S1+2, AIS & Adeno & MET & p.Asp1028His & chr7:116412043 & G & $\mathrm{G} / \mathrm{C}$ & $36 \%$ \\
\hline & left S1+2, MIA & Adeno & EGFR & p.E746-A750 del & chr7:55242465 & AGGAATTAAGAGAAGC & AGGAATTAAGAGAAGC/A & $25 \%$ \\
\hline \multirow[t]{2}{*}{8} & & Adeno & TP53 & p.Arg249Ser & $\operatorname{chr} 17: 7577528$ & GATGGGCCTCCGGTTC & $\begin{array}{l}\text { GATGGGCCTCCGGTTC/ } \\
\text { GATGGGACTCCGGTTC }\end{array}$ & $84 \%$ \\
\hline & & Adeno & RASA1 & p.Gly434Ter & $\operatorname{chr} 5: 86649020$ & G & $\mathrm{G} / \mathrm{T}$ & $78 \%$ \\
\hline \multirow[t]{10}{*}{ right S2 } & right S2 & Adeno & CDKN2A & p.Asp74Tyr & chr9:21971138 & $\mathrm{C}$ & $\mathrm{C} / \mathrm{A}$ & $72 \%$ \\
\hline & & Adeno & FGFR1 & p.Lys436Glu & chr8:38277122 & $\mathrm{T}$ & $\mathrm{T} / \mathrm{C}$ & $58 \%$ \\
\hline & & Adeno & KMT2D & p.Leu4467His & chr12:49425088 & A & $\mathrm{A} / \mathrm{T}$ & $4 \%$ \\
\hline & & small & TP53 & p.Cys242Phe & $\operatorname{chr} 17: 7577553$ & ATGCAGGAACTGT & $\begin{array}{l}\text { ATGCAGGAACTGT/ } \\
\text { ATGAAG GAACTGT }\end{array}$ & $86 \%$ \\
\hline & & small & EP300 & p.Lys1783Arg & chr22:41573063 & A & $\mathrm{A} / \mathrm{G}$ & $48 \%$ \\
\hline & & small & NOTCH1 & p.Glu286Ter & chr9:139413904 & $\mathrm{C}$ & $\mathrm{C} / \mathrm{A}$ & $47 \%$ \\
\hline & left S8 & small & EGFR & p.Phe481Leu & chr7:55227976 & $\mathrm{T}$ & $\mathrm{T} / \mathrm{A}$ & $46 \%$ \\
\hline & & small & NOTCH1 & p.Gln58Ter & chr9:139418400 & G & $\mathrm{G} / \mathrm{A}$ & $45 \%$ \\
\hline & & small & NF1 & p.Arg440Gln & chr17:29533316 & G & G/A & $30 \%$ \\
\hline & & small & PTEN & p.Thr277Ile & chr10:89720679 & $\mathrm{C}$ & $\mathrm{C} / \mathrm{T}$ & $29 \%$ \\
\hline \multirow[t]{5}{*}{9} & & Adeno & EGFR & p.Leu858Arg & chr7:55259515 & TG & TG/GG & $10 \%$ \\
\hline & right S3 & Adeno & ATM & p.Glu1971Lys & chr11:108181035 & G & $\mathrm{G} / \mathrm{A}$ & $7 \%$ \\
\hline & & Adeno & KMT2D & p.Asp1749Glu & chr12:49437723 & A & $\mathrm{A} / \mathrm{C}$ & $4 \%$ \\
\hline & left S9 & Adeno & SOX2 & p.Glu282Val & chr3:181430993 & A & $\mathrm{A} / \mathrm{T}$ & $4 \%$ \\
\hline & & Adeno & KMT2D & p.Asp632Glu & chr12:49445570 & G & $\mathrm{G} / \mathrm{C}$ & $4 \%$ \\
\hline
\end{tabular}




\begin{tabular}{|c|c|c|c|c|c|c|c|c|}
\hline \multirow[t]{6}{*}{10} & & $\mathrm{SCC}$ & CDKN2A & p.Asp108Tyr & chr9:21971036 & $\mathrm{C}$ & $\mathrm{C} / \mathrm{A}$ & $69 \%$ \\
\hline & \multirow{4}{*}{ left S3 } & SCC & CDKN2A & p.Leu104fs & chr9:21971041 & ACGTCCAGCCGCGCC & ACGTCCAGCCGCGCC/A & $69 \%$ \\
\hline & & SCC & NOTCH1 & p.Cys1490Trp & chr9:139399878 & G & $\mathrm{G} / \mathrm{C}$ & $56 \%$ \\
\hline & & SCC & TP53 & p.Arg280Ile & chr17:7577094 & GGTCTCT & GGTCTCT/GGTCTAT & $52 \%$ \\
\hline & & $\mathrm{SCC}$ & SMARCA4 & p.Asp779Tyr & chr19:11123685 & G & $\mathrm{G} / \mathrm{T}$ & $43 \%$ \\
\hline & left S8 & Adeno & EGFR & p.Leu858Arg & chr7:55259515 & TG & $\mathrm{TG} / \mathrm{GG}$ & $29 \%$ \\
\hline \multirow[t]{9}{*}{11} & \multirow{5}{*}{ left S6 } & SCC & TP53 & p.Arg280Thr & chr17:7577094 & GGTCTCT & GGTCTCT/GGTCTGT & $80 \%$ \\
\hline & & SCC & NFE2L2 & p.Leu30Phe & chr2:178098957 & G & G/A & $72 \%$ \\
\hline & & SCC & FGFR3 & p.Ala636Thr & chr4:1807841 & G & G/A & $11 \%$ \\
\hline & & SCC & ERBB2 & p.Asp1144His & chr17:37883959 & G & $\mathrm{G} / \mathrm{C}$ & $4 \%$ \\
\hline & & SCC & CREBBP & p.Leu551Ile & $\operatorname{chr16:3831230}$ & G & $\mathrm{G} / \mathrm{T}$ & $4 \%$ \\
\hline & \multirow{4}{*}{ left S10 } & small & KMT2D & p.Gln3969Leu & chr12:49426582 & $\mathrm{T}$ & $\mathrm{T} / \mathrm{A}$ & $54 \%$ \\
\hline & & small & TP53 & p.Val197Met & chr17:7578256 & $\begin{array}{l}\text { TCCACTCGGATAAGA } \\
\text { TGCTGAGGAGGGG }\end{array}$ & $\begin{array}{l}\text { TCCACTCGGATAAGA } \\
\text { TGCTG AGGAGGGG/ } \\
\text { TCCATTCGGATAAG } \\
\text { ATGCTGAGGAGGGG }\end{array}$ & $18 \%$ \\
\hline & & small & KMT2D & p.Cys $778 \mathrm{fs}$ & chr12:49445134 & A & $\mathrm{A} / \mathrm{AC}$ & $18 \%$ \\
\hline & & small & KMT2D & p.Asp632Glu & chr12:49445570 & G & $\mathrm{G} / \mathrm{C}$ & $5 \%$ \\
\hline \multirow[t]{2}{*}{12} & right S2 & Adeno & RBM10 & p.Tyr573Ter & chrX:47040994 & $\mathrm{C}$ & $\mathrm{C} / \mathrm{G}$ & $43 \%$ \\
\hline & right S3 & Adeno & EGFR & p.Leu858Arg & chr7:55259515 & TG & $\mathrm{TG} / \mathrm{GG}$ & $33 \%$ \\
\hline
\end{tabular}

The entries in bold letters in Case 3 indicate the results from mutation analysis of bronchoscopic specimens, not surgically resected specimens. AF, allele fraction; var., variance; S, segment; SCC, squamous cell carcinoma; Adeno, adenocarcinoma; small, small cell carcinoma; bx, biopsy; chr, chromosome; AIS, adenocarcinoma in situ; MIA, microinvasive adenocarcinoma.

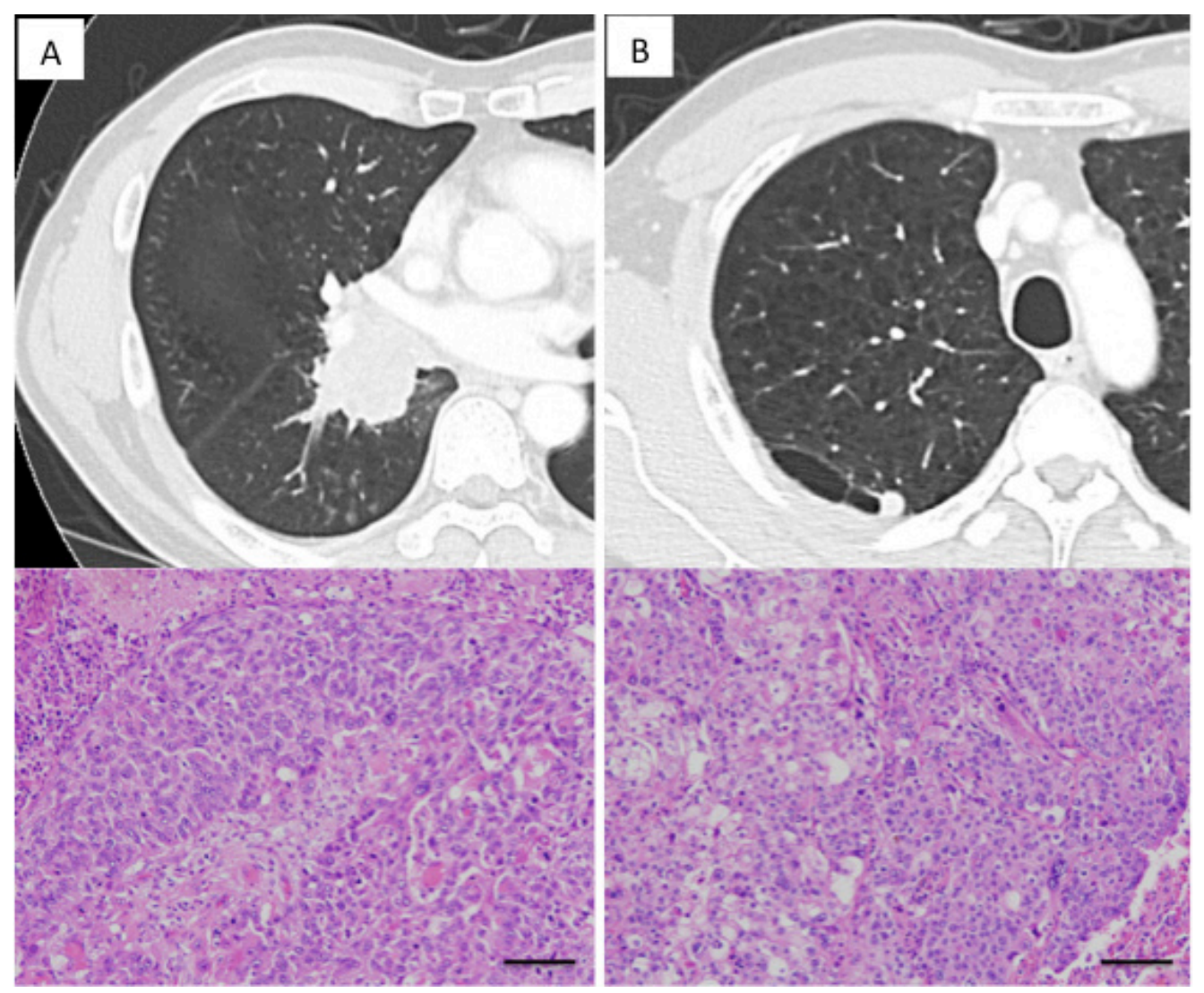

Figure 2: Radiologic findings of lung cancers in Case 1. (A) A large tumor affecting both the middle and lower lobes, diagnosed as squamous cell carcinoma. (B) A small nodular shadow neighboring the bulla was noted in the right upper lobe. Postoperative histological examination revealed it to be squamous cell carcinoma and distinction between primary and metastatic was difficult. Each scale bar indicates $100 \mu \mathrm{m}$. 


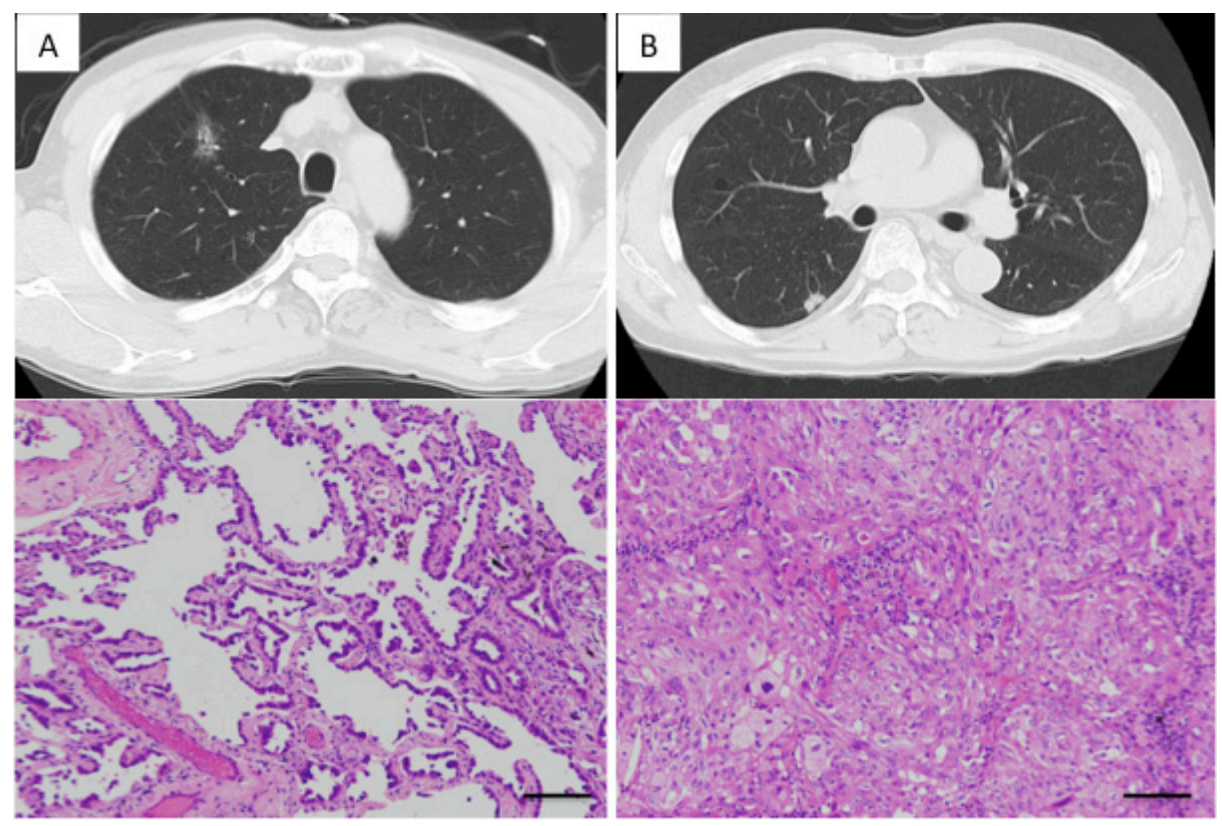

Figure 3: Radiologic findings of the lung cancers in Case 3. (A) Right upper lobe shadow: A shadow, primarily ground glass opacity and accompanied by partially solid consolidation. Histologically, it was diagnosed as lepidic predominant adenocarcinoma. (B) Right lower lobe shadow: Nodular shadow adjacent to the pleura. Histologically, it was diagnosed as solid predominant adenocarcinoma. Each scale bar indicates $100 \mu \mathrm{m}$.
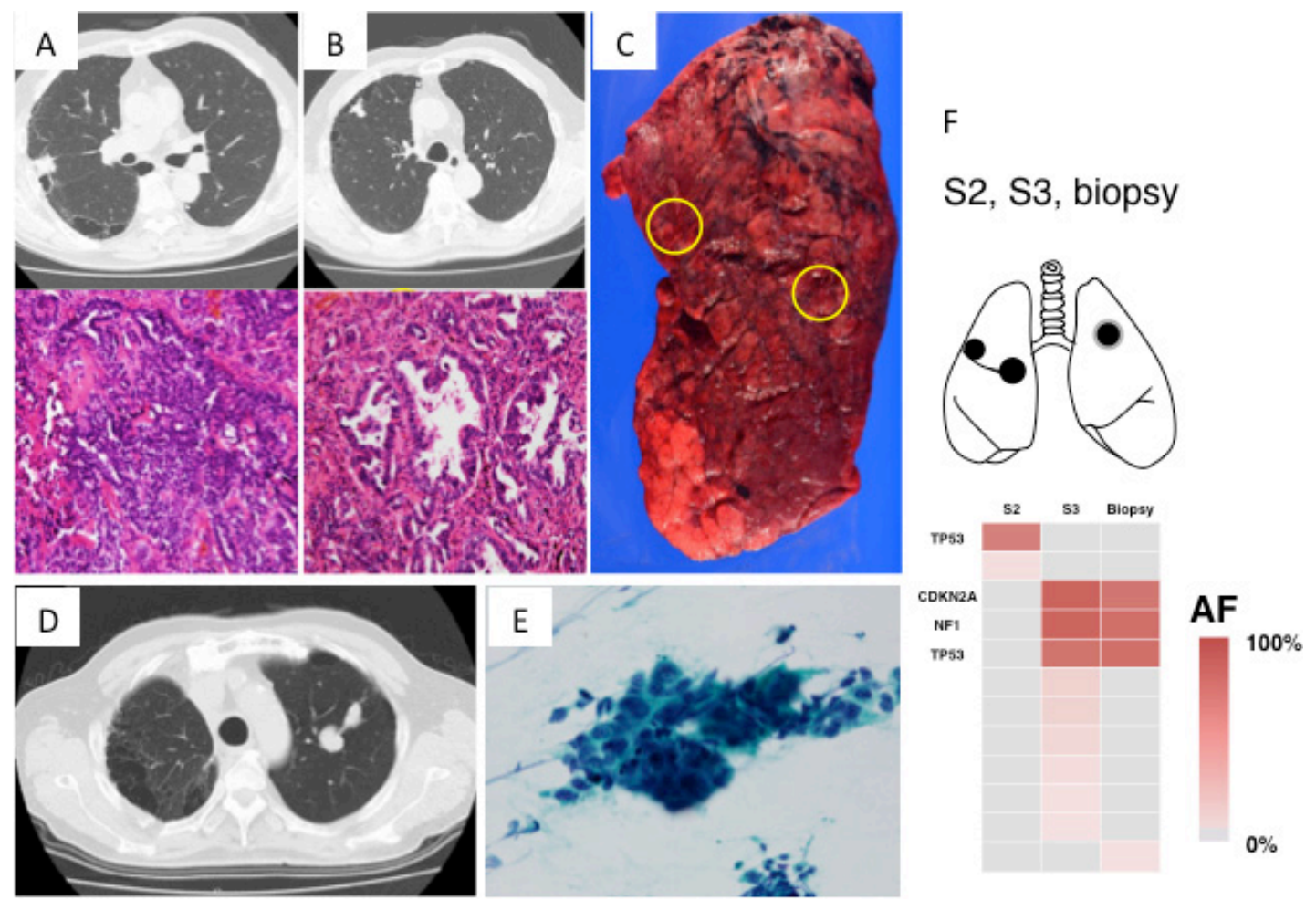

Figure 4: Radiologic, pathologic and genomic findings of the lung cancer in Case 3. (A) Lung cancer in right S2: solid predominant adenocarcinoma. (B) Lung cancer in right S3: papillary predominant adenocarcinoma. (C) Macroscopic view of the S2 and S3 lung cancers. Yellow circle indicates the locations of the cancers. (D) A solitary lung lesion newly developing in the left lung postoperatively. Radiologically, distinguishing between primary or metastatic cancer was difficult. (E) Histopathology of the bronchoscopic biopsy specimen: poorly differentiated adenocarcinoma. (F) Heat map of the gene mutations of 3 lung tumors. Mutation differed between right S2 and S3 tumors, but the significant mutations in right $\mathrm{S} 3$ tumor were homologous to those in left $\mathrm{S} 1+2$ tumor. S, segment; AF allele fraction. 


\section{DISCUSSION}

In cases of multiple lung cancers, clinical distinction between primary and metastatic tumors is sometimes difficult, making treatment selection challenging. Thus, we conducted lung cancer mutation analysis by targeted deep sequencing and found that mutations in individual lung cancers could serve as a clonal marker, allowing distinction of the clonality of individual tumors.

In Case 1, pre-operative distinction between primary and metastatic tumors on the basis of clinical course, diagnostic imaging, and pathological examination was difficult. Even experienced radiologists interpreted the PET and CT findings to indicate intrapulmonary metastasis, although the true diagnosis was double primary cancers. Thus, it is possible that certain multiple primary lung cancers are misdiagnosed as metastatic cancer based on pathology and radiology [7]. Our novel approach may help resolve the current dilemma of misdiagnosis in the clinical setting.

In Case 3, the mutation found in the right $\mathrm{S} 3$ tumor was consistent with that in the left $\mathrm{S} 1+2$ tumor, demonstrating that this was a case of lung cancers from the same clone (i.e., intrapulmonary metastasis). Consistency in multiple mutations, with complete consistency observed even in the position and patterns of base-pair substitutions, cannot be an accidental phenomenon.
Although inconsistency between the two tumors was noted in mutations of less than $10 \%$ of the allele fraction, this can be explained by tumor heterogeneity. In general, cancers are composed of populations of cells with distinct molecular and phenotypic features, a phenomenon termed intratumor heterogeneity $[8,9]$. Intratumor heterogeneity, associated with heterogeneous protein function, may foster tumor adaptation, phenotypic aggravation, and/ or therapeutic failure through Darwinian selection $[10,11]$. In contrast, a pivotal driver mutation serves as the trigger of clonal expansion and is estimated to be retained homogenously within the tumors of the same clone [11-14], regardless of whether they are intra- or extrapulmonary lesions, such as the skin metastasis in Case 3. These events can be explained by the "trunk and branch" mutation models, i.e., early somatic events that drive tumor growth or maintenance in early clonal progenitors are represented within the "trunk" of the tumor $[8,9]$. Such trunk somatic aberrations, present at the early stages of tumor development, are likely to be ubiquitous events occurring at all sites of disease. In contrast, later somatic events that occur following branched separation of subclones represent heterogeneous events. Such subclonal heterogeneity may be spatially separated between regions of the same tumor or its metastatic sites [8-10]. In this context, clonally dominant "trunk" somatic aberrations are important clonal markers. Primary and metastatic

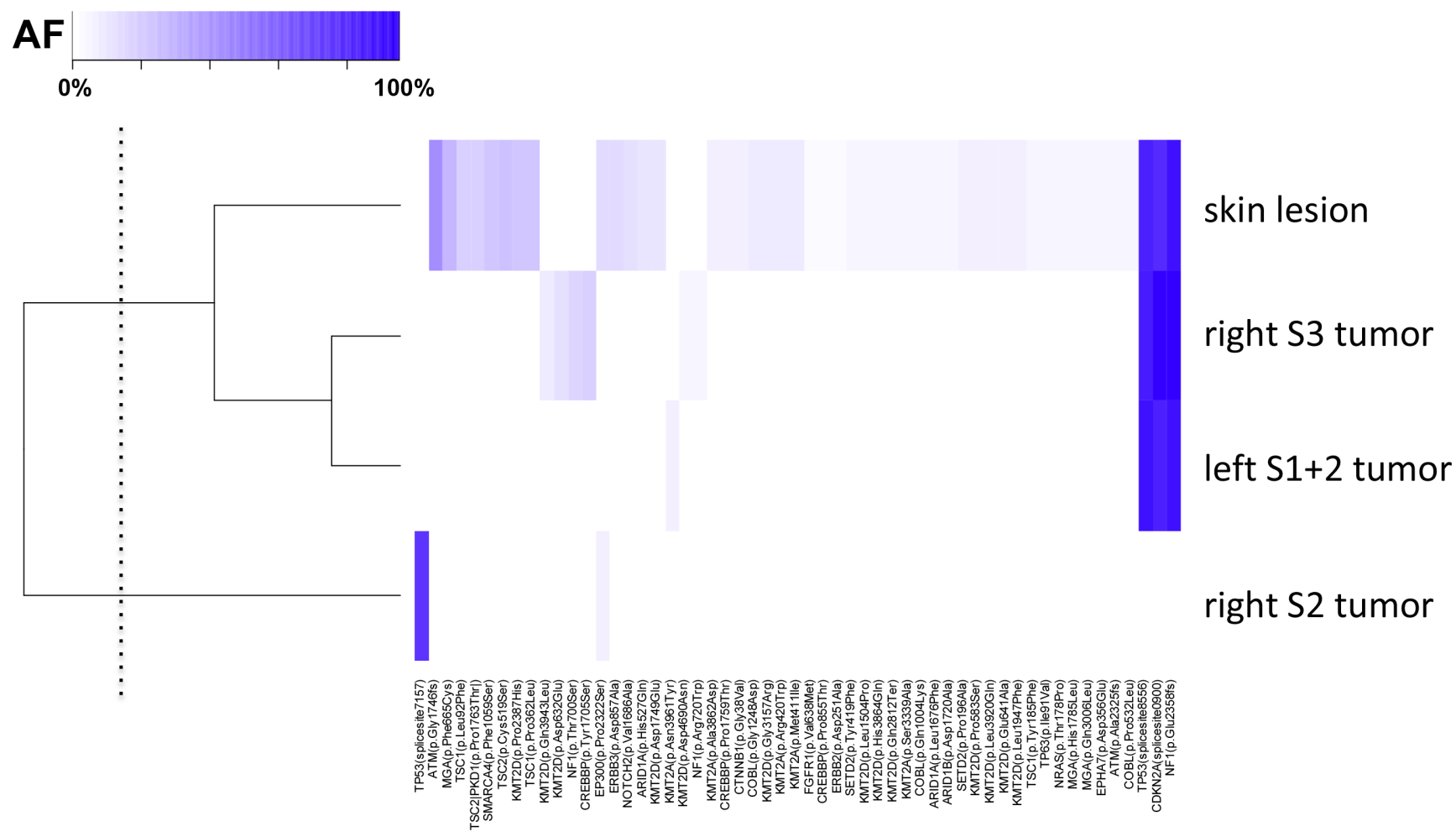

Figure 5: Cluster analysis of the point mutations in Case 3. The mutation data were standardized and presented as a heat map. The skin lesion harbored the common driver mutations with right S3 and left S1+2 cancers. By the cluster analysis, the lesions that share a similar pattern of allele fraction were identified. Unsupervised hierarchial clustering was used to group correlated lesions, with the dendrogram threshold level to establish two clusters indicated on the y-axis (dotted line). Column at bottom of heatmap shows the mutated genes and amino acid changes. 
tumors can be discriminated by determining whether such ubiquitous mutations are identical.

It can be relatively easy to diagnose multicentric primary lung cancers when their histological types are different. However, if they are of the same histological type, it is often difficult to discriminate multiple primary lung cancers from intrapulmonary metastasis. Particularly in cases such as Case 1 (multiple tumors classified as squamous cell carcinoma), making a distinction by pathology alone is difficult. A bronchoscopic examination involves a biopsy of part of the tumor, preventing assessment of the full pathological profile and occasionally making it difficult to distinguish between primary and metastatic tumors. In our cases, the bronchoscopically biopsied specimens were very small (about $1 \mathrm{~mm}^{3}$ each), but mutation analysis was possible, allowing comparative analysis of the mutations. Even when morphological and immunohistological features are nonhomogeneous among different parts of the tumors, the driver mutation serving as the trunk is prevalently retained within the tumors of the same clone [11-14]. Therefore, distinction of clonality based on mutation analysis is suggested to be more specific and definitive than histological distinction. Furthermore, based on the hierarchical clustering results, we could subclassify the four lesions of clonal diversity into two distinct groups. Thus, cluster analysis is helpful for evaluating the clonal progression of the cancer among the multiclonal cancer lesions. Even if part of the mutation profile is matched between two tumors to some degree, these tumors can be presumed to be primary or metastatic by performing cluster analysis to estimate the genetic distance between them.

Detterbeck et al. summarized clinical and pathologic criteria to distinguish second primary versus metastatic tumors; they concluded that few features are definitive and that it is difficult to define criteria that conclusively establish that the tumors are identical; finding similarities is not sufficient $[15,16]$. In our method, when clonally dominant mutations with high allele fractions are also completely identical at the base-pair level, they are clearly defined as the same clone, as we showed in the presentation of Case 3. In other words, comparison of "trunk" mutations could yield definitive criteria. If "trunk" mutations are homologous, it would be easy to determine whether two tumors are the same.

In mutation analysis, many previous studies assessed particular mutations to define clonality, assuming that a match of a few (one to five) markers defines a single clone whereas a difference defines separate cancers [17-21]. However, although one to five mutations that are not "trunk" mutations are compared, their differences are not significant as the basis for discriminating between primary and metastatic tumors, and this characterization is associated with potential misclassification. According to our method, comprehensive mutation analysis is first performed to identify "trunk" mutations of each cancer, which are then compared to define their clonality. These criteria are not so much suggestive as definitive and reliable. In addition, they are relatively simple because they are measurable by next-generation sequencing alone. Moreover, the decision criteria are generally clear and intuitive. Thus, we expect that our method will be widely adopted as a standard diagnostic method in daily clinical practice in the future.

When treatment methods are selected for multiple lung cancers, it is necessary to consider which of the multiple cancers will affect the prognosis of patients most. In Case 2, malignant pleural effusion was noted, and the disease was diagnosed as stage IV. The factor responsible for progression to such an advanced stage was identified as the S6 adenocarcinoma with pleural invasion between the two adenocarcinomas. Therefore, treatment for this case will target the S6 adenocarcinoma. At present, molecular-targeted therapies of lung cancer are confined to those targeting EGFR and $A L K$ mutations. The development of novel moleculartargeted therapies would enable postoperative adjuvant chemotherapy and recurrent tumor treatment tailored to the features of mutations in individual cancers. Thus, in cases of multiple lung cancers, checking mutations is also important based on selection of the most effective medical treatments.

In conclusion, when dealing with synchronous or metachronous multiple lung cancers, checking the differences in the mutation profile among multiple tumors will clarify the clonal origin of the tumors and enable distinction between primary and metastatic tumors with high specificity, even in cases where pathological distinction is not possible. Thus, treatment tailored to the features of individual cases will be possible. Furthermore, if mutation analysis is performed for bronchoscopically collected specimens, pre-operative diagnosis will be possible, and the treatment strategy for multiple lung cancers may thus be devised rationally.

\section{MATERIALS AND METHODS}

\section{Patients and sample preparation}

The study involved 12 patients who underwent surgery in our department for synchronous multiple lung cancers between July 2014 and March 2016. These patients provided written informed consent for the genetic research studies, which were performed in accordance with protocols approved by the Institutional Review Board at our hospital.

The serial section of formalin-fixed, paraffinembedded (FFPE) tissue was stained with hematoxylineosin and then micro-dissected using an ArcturusXT lasercapture microdissection system (Thermo Fisher Scientific, Tokyo, Japan). DNA was extracted using the QIAamp DNA FFPE Tissue Kit (Qiagen). FFPE DNA quality was checked using primers for ribonuclease $\mathrm{P}$ (RNase P) locus. A peripheral blood sample was drawn from each patient just prior to surgery. Buffy coat were isolated following centrifugation and DNA was extracted from buffy coat using the QIAamp DNA Blood Mini Kit (Qiagen, Tokyo, Japan). 


\section{Targeted deep sequencing and data analysis}

A panel targeting the exon of 53 lung cancer-associated genes (see Supplementary Table 1) was established to perform targeted sequencing. We searched the literature and selected these genes based on the following criteria: (a) genes often involved in lung cancer reported TCGA $[22,23]$ and other projects [24-28] or (b) genes frequently mutated in lung cancer from the COSMIC database (http://cancer.sanger.ac.uk/ cancergenome/projects/cosmic). The primer design for the targeted sequencing was performed by Ion AmpliSeq designer software (Thermo Fisher Scientific), as we previously reported $[29,30]$. Sequencing libraries were prepared using Ion AmpliSeq Library kit (Thermo Fisher Scientific), according to the manufacturer's instruction. After barcode ligation using Ion Xpress Barcode Adapters kit (Thermo Fisher Scientific), library samples were purified using Agencourt AMPure XP reagent (Beckman Coulter, Tokyo, Japan) and subsequently quantified using Ion Library Quantitation Kit (Thermo Fisher Scientific). The libraries were templated with the Ion PI Template OT2 200 Kit v3 (Thermo Fisher Scientific). Sequencing was carried out on an Ion Proton (Ion Torrent) using the Ion PI Sequencing 200 Kit v3.

The sequence data were processed using standard Ion Torrent Suite Software running on the Torrent Server. Raw signal data were analyzed using Torrent Suite version 4.0. The pipeline included signaling processing, base calling, quality score assignment, read alignment to the human genome 19 reference (hg19), quality control of mapping and coverage analysis. Following data analysis, annotation of single nucleotide variants, insertions and deletions was performed by the Ion Reporter Server System (Thermo Fisher Scientific), and lymphocytes from peripheral blood DNA were used as a control to detect any variants (TumorNormal pair analysis). Sequence data were visually confirmed with the Integrative Genomics Viewer.

Unsupervised hierarchical clustering was used to identify potential distinct subgroups among the multiclonal lesions based on the genetic profiling. The genetic informations were standardized, clustered and visualized with the CLUSTER and TREEVIEW programs.

\section{Abbreviations}

PCR: polymerase chain reaction; CT: computed tomography; PET: positron emission tomography; S: segment.

\section{Authors' contributions}

TG and YH contributed equally to this work. TG and YH wrote the manuscript. TG, TN, DS and YY performed surgery. TO performed the pathological examination. YH, $\mathrm{KA}, \mathrm{TG}, \mathrm{TN}, \mathrm{YY}, \mathrm{HM}$ and $\mathrm{MO}$ participated in the genomic analyses. $\mathrm{MO}$ and $\mathrm{YH}$ were involved in the final editing. All authors have read and approved the final manuscript.

\section{ACKNOWLEDGMENTS}

The authors greatly appreciate Takuro Uchida, Hidetoshi Shigetomo and Yumi Kubota for helpful scientific discussions.

\section{CONFLICTS OF INTEREST}

The authors declare that they have no conflicts of interest.

\section{FUNDING}

This study was supported by a Grant-in-Aid for Genome Research Project from Yamanashi Prefecture (Y.H. and M.O.) and the grant from The YASUDA Medical Foundation (Y.H.).

\section{REFERENCES}

1. Matsuzoe D, Hideshima T, Ohshima K, Kawahara K, ShirakusaT, Kimura A. Discrimination of double primary lung cancer from intrapulmonary metastasis by p53 gene mutation. Br J Cancer. 1999; 79:1549-1552.

2. Mitsudomi T, Yatabe $\mathrm{Y}$, Koshikawa $\mathrm{T}$, Hatooka $\mathrm{S}$, Shinoda M, Suyama M, Sugiura T, OgawaM, Takahashi T. Mutations of the P53 tumor suppressor gene as clonal marker for multiple primary lung cancers. J Thorac Cardiovasc Surg. 1997; 114:354-360.

3. Ono K, Sugio K, Uramoto H, Baba T, Ichiki Y, TakenoyamaM, Hanagiri T, OyamaT, Yasumoto K. Discrimination of multiple primary lung cancers from intrapulmonary metastasis based on the expression of four cancer-related proteins. Cancer. 2009; 115:3489-3500.

4. van der Sijp JR, van Meerbeeck JP, Maat AP, Zondervan PE, Sleddens HF, van Geel AN, EggermontAM, Dinjens WN. Determination of the molecular relationship between multiple tumors within one patient is of clinical importance. J Clin Oncol. 2002; 20:1105-1114.

5. van Oijen MG, Leppers Vd Straat FG, TilanusMG, Slootweg PJ. The origins of multiple squamous cell carcinomas in the aerodigestive tract. Cancer. 2000; 88:884-893.

6. Shimizu S, Yatabe Y, Koshikawa T, Haruki N, Hatooka S, Shinoda M, Suyama M, Ogawa M, Hamajima N, Ueda R, TakahashiT, Mitsudomi T. High frequency of clonally related tumors in cases of multiple synchronous lung cancers as revealed by molecular diagnosis. Clin Cancer Res. 2000; 6:3994-3999.

7. Nakajima J, Furuse A, Oka T, KohnoT, Ohtsuka T. Excellent survival in a subgroup of patients with intrapulmonary metastasis of lung cancer. Ann Thorac Surg. 1996; 61:158 162; discussion 162-153.

8. Yap TA, Gerlinger M, Futreal PA, PusztaiL, Swanton C. Intratumor heterogeneity: seeing the wood for the trees. Sci Transl Med. 2012; 4:127ps110. 
9. Swanton C. Intratumor heterogeneity: evolution through space and time. Cancer Res. 2012; 72:4875-4882.

10. Gerlinger $\mathrm{M}$, Rowan AJ, Horswell S, Larkin J, Endesfelder D, Gronroos E, Martinez P, Matthews N, Stewart A, Tarpey P, Varela I, Phillimore B, Begum S, et al. Intratumor heterogeneity and branched evolution revealed by multiregion sequencing. N Engl J Med. 2012; 366:883-892.

11. Goto T, Hirotsu Y, Mochizuki H, Nakagomi T, Oyama T, AmemiyaK, Omata M. Stepwise addition of genetic changes correlated with histological change from "welldifferentiated" to "sarcomatoid" phenotypes: a case report. BMC Cancer. 2017; 17:65.

12. de Bruin EC, McGranahan N, Mitter R, Salm M, Wedge DC, Yates L, Jamal-Hanjani M, Shafi S, Murugaesu N, Rowan AJ, Gronroos E, Muhammad MA, Horswell S, et al. Spatial and temporal diversity in genomic instability processes defines lung cancer evolution. Science. 2014; 346:251-256.

13. Yatabe Y, MatsuoK, Mitsudomi T. Heterogeneous distribution of EGFR mutations is extremely rare in lung adenocarcinoma. J Clin Oncol. 2011; 29:2972-2977.

14. Zhang J, Fujimoto J, Zhang J, Wedge DC, Song X, Zhang J, Seth S, Chow CW, Cao Y, Gumbs C, Gold KA, Kalhor N, Little L, et al. Intratumor heterogeneity in localized lung adenocarcinomas delineated by multiregion sequencing. Science. 2014; 346:256-259.

15. Detterbeck FC, Franklin WA, Nicholson AG, Girard N, Arenberg DA, Travis WD, Mazzone PJ, Marom EM, Donington JS, Tanoue LT, Rusch VW, Asamura H, RamiPorta R, et al. The IASLC Lung Cancer Staging Project: Background Data and Proposed Criteria to Distinguish Separate Primary Lung Cancers from Metastatic Foci in Patients with Two Lung Tumors in the Forthcoming Eighth Edition of the TNM Classification for Lung Cancer. J Thorac Oncol. 2016; 11:651-665.

16. Detterbeck FC, Nicholson AG, Franklin WA, Marom EM, Travis WD, Girard N, Arenberg DA, Bolejack V, Donington JS, Mazzone PJ, Tanoue LT, Rusch VW, Crowley J, et al. The IASLC Lung Cancer Staging Project: Summary of Proposals for Revisions of the Classification of Lung Cancers with Multiple Pulmonary Sites of Involvement in the Forthcoming Eighth Edition of the TNM Classification. J Thorac Oncol. 2016; 11:639-650.

17. Chang YL, Wu CT, Lin SC, Hsiao CF, JouYS, Lee YC. Clonality and prognostic implications of p53 and epidermal growth factor receptor somatic aberrations in multiple primary lung cancers. Clin Cancer Res. 2007; 13:52-58.

18. Chung JH, Choe G, Jheon S, Sung SW, Kim TJ, Lee KW, LeeJH, Lee CT. Epidermal growth factor receptor mutation and pathologic-radiologic correlation between multiple lung nodules with ground-glass opacity differentiates multicentric origin from intrapulmonary spread. J Thorac Oncol. 2009; 4:1490-1495.
19. Girard N, Deshpande C, Azzoli CG, Rusch VW, Travis WD, LadanyiM, Pao W. Use of epidermal growth factor receptor/ Kirsten rat sarcoma 2 viral oncogene homolog mutation testing to define clonal relationships among multiple lung adenocarcinomas: comparison with clinical guidelines. Chest. 2010; 137:46-52.

20. Takamochi K, Oh S, MatsuokaJ, Suzuki K. Clonality status of multifocal lung adenocarcinomas based on the mutation patterns of EGFR and K-ras. Lung Cancer. 2012; 75:313-320.

21. Wang X, Wang M, MacLennan GT, Abdul-Karim FW, Eble JN, Jones TD, Olobatuyi F, Eisenberg R, Cummings OW, Zhang S, Lopez-Beltran A, Montironi R, Zheng S, et al. Evidence for common clonal origin of multifocal lung cancers. J Natl Cancer Inst. 2009; 101:560-570.

22. Cancer Genome Atlas Research Network. Comprehensive molecular profiling of lung adenocarcinoma. Nature. 2014; 511:543-550.

23. Cancer Genome Atlas Research Network. Comprehensive genomic characterization of squamous cell lung cancers. Nature. 2012; 489:519-525.

24. Clinical Lung Cancer Genome Project (CLCGP); Network Genomic Medicine (NGM). A genomics-based classification of human lung tumors. Sci Transl Med. 2013; 5:209ra153.

25. Rudin CM, Durinck S, Stawiski EW, Poirier JT, Modrusan Z, Shames DS, Bergbower EA, Guan Y, Shin J, Guillory J, Rivers CS, Foo CK, Bhatt D, et al. Comprehensive genomic analysis identifies SOX2 as a frequently amplified gene in small-cell lung cancer. Nat Genet. 2012; 44:1111-1116.

26. Peifer M, Fernandez-Cuesta L, Sos ML, George J, Seidel D, Kasper LH, Plenker D, Leenders F, Sun R, Zander T, Menon R, Koker M, Dahmen I, et al. Integrative genome analyses identify key somatic driver mutations of small-cell lung cancer. Nat Genet. 2012; 44:1104-1110.

27. Imielinski M, Berger AH, Hammerman PS, Hernandez B, Pugh TJ, Hodis E, Cho J, Suh J, Capelletti M, Sivachenko A, Sougnez C, Auclair D, Lawrence MS, et al. Mapping the hallmarks of lung adenocarcinoma with massively parallel sequencing. Cell. 2012; 150:1107-1120.

28. Govindan R, Ding L, Griffith M, Subramanian J, Dees ND, Kanchi KL, Maher CA, Fulton R, Fulton L, Wallis J, Chen K, Walker J, McDonald S, et al. Genomic landscape of non-small cell lung cancer in smokers and neversmokers. Cell. 2012; 150:1121-1134.

29. Hirotsu Y, Nakagomi H, Sakamoto I, Amemiya K, Oyama T, MochizukiH, Omata M. Multigene panel analysis identified germline mutations of DNA repair genes in breast and ovarian cancer. Mol Genet Genomic Med. 2015; 3:459-466.

30. Hirotsu Y, Nakagomi H, Sakamoto I, Amemiya K, MochizukiH, Omata M. Detection of BRCA1 and BRCA2 germline mutations in Japanese population using next-generation sequencing. Mol Genet Genomic Med. 2015; 3:121-129. 